\title{
IncRNA LOC339524 inhibits the proliferation of bladder cancer cells by targeting the miR-875-5p/COPS7A signaling axis
}

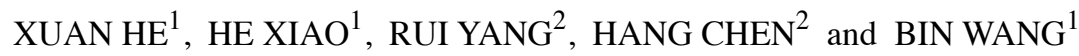 \\ ${ }^{1}$ Department of Oncology, Daping Hospital, Army Medical University, Chongqing 400042; \\ ${ }^{2}$ Department of Cell Biology and Genetics, Chongqing Medical University, Chongqing 400016, P.R. China
}

Received February 18, 2021; Accepted July 21, 2021

DOI: $10.3892 / \mathrm{etm} .2021 .10636$

\begin{abstract}
It has been reported that long non-coding RNAs (lncRNAs) play a crucial role in the progression of various types of cancer. The role of numerous lncRNAs in a variety of cancer types has been investigated. However, the underlying mechanisms of the majority of lncRNAs in bladder cancer (BCa) remain to be elucidated. In the present study, abnormally expressed lncRNAs in $\mathrm{BCa}$ and para-carcinoma tissues were identified through screening the Cancer RNA-Seq Nexus database and were validated using reverse transcription-quantitative PCR. It was found that LOC339524 expression levels were markedly downregulated in BCa tissues and cells (J82, T24, UM-UC-3 and 5637). LOC339524 overexpression was revealed to suppress the proliferation of BCa cells. LOC339524 was also discovered to act as a sponge for microRNA (miR)-875-5p, as identified using dual luciferase reporter assays and biotin pull-down analysis. LOC339524 downregulated the expression of miR-875-5p and knockdown of miR-875-5p expression inhibited the proliferation of bladder cancer cells. In addition, COP9 signalosome subunit 7A (COPS7A) was identified to be the target gene of miR-875-5p and COPS7A expression level was upregulated following LOC339524 overexpression. IncRNA LOC339524 was proposed to function as a competitive endogenous RNA to facilitate the expression of COPS7A by binding to miR-875-5p. In conclusion, the findings of the present study suggested that LOC339524 may inhibit cell proliferation in BCa by targeting the miR-875-5p/COPS7A signaling axis.
\end{abstract}

\section{Introduction}

According to a recent study, bladder cancer $(\mathrm{BCa})$ is the most common type of malignancy of the urinary system and poses a major threat to human health worldwide, 573,278 new

Correspondence to: Dr Bin Wang, Department of Oncology, Daping Hospital, Army Medical University, 10 Changjiang Road, Daping, Yuzhong, Chongqing 400042, P.R. China

E-mail: wangjianlinbin@163.com

Key words: LOC339524, bladder cancer, microRNA-875-5p, proliferation cases of bladder cancer and 212,536 mortalities from bladder cancer occurred in 2021 (1). Surgery and chemotherapy are the predominant treatment strategies used for $\mathrm{BCa}$; however, the treatment efficacy is far from satisfactory (2). Thus, novel therapeutic strategies for this disease are required.

Long non-coding RNAs (IncRNAs) are a class of non-coding RNA of $>200$ nucleotides in length $(3,4)$. As they are generated via RNA polymerase II-mediated transcription, IncRNAs have been identified to play a crucial role in multiple types of cancer, including colon and non-small cell lung cancer, hepatocellular carcinoma and breast cancer (5-8). According to recent studies, lncRNAs can inhibit or promote the expression of certain genes at the transcriptional or post-transcriptional level $(9,10)$. For instance, IncRNA small nucleolar RNA host gene 3 (SNHG3) is significantly upregulated in $\mathrm{BCa}$ tissues and associated with reduced overall survival rates of BCa patients. Silencing of SNHG3 expression suppresses BCa cell proliferation and metastasis by increasing miR-515-5p expression (11). IncRNA KCNQ1 opposite strand/antisense transcript 1 is notably upregulated in $\mathrm{BCa}$ tissues and promote the progression of $\mathrm{BCa}$ through by modulating miR-218-5p expression and the activity of Heparan Sulfate-Glucosamine 3-Sulfotransferase 3B1 (12).

In the present study, abnormally expressed lncRNAs in $\mathrm{BCa}$ tissues and adjacent normal tissues were examined using the Cancer RNA-Seq Nexus (CRN) database, with a particular focus was on the IncRNAs that were lowly expressed in $\mathrm{BCa}$ and had not yet been reported, which may have more research value. To the best of our knowledge, as the role of lncRNA LOC339524 in $\mathrm{BCa}$ has not been studied before, it was selected for subsequent analysis. The aims of the present study were to investigate the expression of IncRNA LOC339524 in BCa, evaluate the effect of IncRNA LOC339524 on regulating the malignant phenotypes of $\mathrm{BCa}$ cells in vitro and explore the mechanism that underlies IncRNA LOC339524 in BCa progression.

\section{Materials and methods}

Patient studies. A total of 52 clinical samples (26 BCa tissues and 26 para-carcinoma tissues) were collected from 26 patients (male, $\mathrm{n}=17$ and female, $\mathrm{n}=9$; age range, 43-67 years) with BCa who underwent surgery at The Third Affiliated Hospital, Army Medical University (Chongqing, China) between September 2017 and February 2019. The tissues were immediately cut into small sections and transferred to liquid 
nitrogen $\left(-196^{\circ} \mathrm{C}\right)$ for use in further experiments. The tissues were all pathologically identified as $\mathrm{BCa}$ or para-carcinoma tissues. Exclusion criteria included a prior history of cancer, chemotherapy or radiotherapy prior to undergoing surgery or a lack of written informed consent. The present study was approved by the Ethics Committee of The Third Affiliated Hospital, Army Medical University. All patients were informed of the study protocol and signed informed consent forms prior to participation.

Cell lines and culture. Human BCa cell lines (J82, T24, UM-UC-3 and 5637) and the normal bladder epithelial cell line, SV-HUC-1, were purchased from Shanghai Cell Bank of Chinese Academy of Sciences. J82, T24 and 5637 cells were routinely cultured in RPMI-1640 medium (HyClone; Cytiva) supplemented with 10\% FBS (Gibco; Thermo Fisher Scientific, Inc.). The UM-UC-3 and SV-HUC-1 were routinely cultured in DMEM (HyClone; Cytiva) supplemented with 10\% FBS. All cells were maintained in an incubator at $37^{\circ} \mathrm{C}$ with $5 \% \mathrm{CO}_{2}$. For $5 \mu$ 5-Aza-2'-deoxycytidine (Sigma-Aldrich; Merck KGaA) treatment, the 5-Aza-2'-deoxycytidine compound was dissolved in DMSO and diluted in cell culture medium to $2 \mu \mathrm{mol} / 1$. Cells were cultured in the cell culture supplemented with DMSO at $37^{\circ} \mathrm{C}$ for three consecutive days as the vehicle-treated cells.

Reverse transcription-quantitative PCR (RT-qPCR). Total RNA was extracted from the tissues and cells using RNAiso Plus (Takara Bio, Inc.). Total RNA was reverse transcribed into cDNA using a PrimeScript RT Master mix (Takara Bio, Inc.) according to the manufacturer's protocol. The following thermocycling conditions were used for reverse transcription: $16^{\circ} \mathrm{C}$ for $30 \mathrm{~min}, 42^{\circ} \mathrm{C}$ for $30 \mathrm{~min}$ and $85^{\circ} \mathrm{C}$ for $5 \mathrm{~min}$. qPCR was subsequently performed using SYBR Premix Ex Taq kit (Takara Bio, Inc.). The thermocycling conditions for qPCR were: $95^{\circ} \mathrm{C}$ for $2 \mathrm{~min}, 40$ cycles of $95^{\circ} \mathrm{C}$ for $15 \mathrm{sec}$ and $60^{\circ} \mathrm{C}$ for $30 \mathrm{sec}$. The primers used for the qPCR are listed in Table SI. GAPDH was selected as the internal reference gene for mRNA, while U6 was selected as the internal reference gene for the normalization of microRNA (miRNA/miR) expression. The $2^{-\Delta \Delta C q}$ method was used for analyzing the relative gene expression (13).

Cell transfection. LOC339524 plasmid (pcDNA3.0), miR-875-5p mimic (5'-UAUACCUCAGUUUUAUCAGGU G-3'), miR-875-5p inhibitor (5'-CACCUGAUAAAACUG AGGUAUA-3'), mimic-NC (5'-UUCUCCGAACGUGUC ACGUTT-3'), inhibitor-NC (5'-CAGUACUUUUGUGUA GUACAA-3') and NC empty plasmid (pcDNA3.0, control for LOC339524) were obtained from Shanghai GeneChem Co., Ltd. The cells were seeded into 6-well plates at a density of $2 \times 10^{5}$ cells/well and prior to transfection, were washed with PBS. Then, $40 \mathrm{nM}$ plasmid, mimic, inhibitor, respective NC or $2 \mu \mathrm{l}$ Lipofectamine ${ }^{\circledR} 2000$ (Invitrogen; Thermo Fisher Scientific, Inc.) were individually diluted in $50 \mu 1$ Opti-MEM (Invitrogen; Thermo Fisher Scientific, Inc.). Following $20 \mathrm{~min}$ of incubation, plasmid/oligonucleotide Opti-MEM solution and Lipofectamine 2000 Opti-MEM solution were mixed and incubated at $4^{\circ} \mathrm{C}$ for a further $20 \mathrm{~min}$. The complex was then added to each well. The cells were collected for subsequent experimentation following $48 \mathrm{~h}$ of further culture.
Cell Counting Kit (CCK)-8 assay. Following transfection for $48 \mathrm{~h}$, cells were seeded into 96 -well plates at a density of $1 \times 10^{3}$ cells/well (six replicates/group). Following the incubation at $37^{\circ} \mathrm{C}$ for $24 \mathrm{~h}$, $10 \mu \mathrm{l}$ CCK-8 reagent (Dojindo Molecular Laboratories, Inc.) was added/well and incubated at $37^{\circ} \mathrm{C}$ for $3 \mathrm{~h}$. The absorbance of each well was measured at a wavelength of $450 \mathrm{~nm}$ using a spectrophotometer (BioTek Instruments, Inc.). The data were collected for 5 days and the experiment was repeated four times.

Colony formation assay. Following transfection for $48 \mathrm{~h}$, cells were seeded into 6 -well plates at a density of $1 \times 10^{3}$ cells/well. Following incubation at $37^{\circ} \mathrm{C}$ for 12 days, the colonies in each group were fixed with $4 \%$ paraformaldehyde at room temperature for $30 \mathrm{~min}$ and stained with $0.1 \%$ crystal violet at room temperature for $30 \mathrm{~min}$. The colonies were visualized and counted. The experiment was repeated four times.

Measurement of DNA content. Following transfection for $48 \mathrm{~h}$, cells were harvested $\left(1,500 \mathrm{x} \mathrm{g} ; 10 \mathrm{~min} ; 4^{\circ} \mathrm{C}\right)$ and suspended in propidium iodide (RNaseA, $10 \mathrm{mg} / \mathrm{l}$; Beyotime Institute of Biotechnology) at $4^{\circ} \mathrm{C}$ for $40 \mathrm{~min}$. Flow cytometric analysis (FACSCalibur flow cytometer; BD Biosciences) was performed to assess the relative DNA content in T24 and 5637 cells with a CellQuest Pro system software package (version 5.1; BD Biosciences).

Bioinformatics analysis. Expression profiles for LOC339524 were obtained from the Cancer RNA-seq Nexus (CRN) database (14) and the Gene Expression Profiling Interactive Analysis (GEPIA) database (http://gepia.cancer-pku.cn/index. html) (15). The DIANA TOOLS database (http://diana.imis. athena-innovation.gr) (16) was used to predict the target miRNA of LOC339524. The MethHC database (https://awi. cuhk.edu.cn/ MethHC/methhc_2020/php/index.php) (17) was used to determine the degree of methylation of the LOC339524 promoter in $\mathrm{BCa}$.

Dual luciferase reporter assay. The binding sites between LOC339524 and miR-875-5p were predicted using the DIANA TOOLS database (http://diana.imis.athena-innovation.gr). The wild-type(WT) LOC339524-WT luciferase plasmid containing the binding sites for miR-875-5p and the LOC339524-mutant (MUT) plasmid containing the mutated binding sites were constructed by Changzhou Ruibo Bio-Technology Co., Ltd. The binding sites between miR-875-5p and the COPS7A 3'-untranslated region (3'-UTR) were predicted using miRWalk (http://zmf.umm.uni-heidelberg.de/apps/zmf/mirwalk2) and TargetScan (http://www.targetscan.org) databases. The COPS7A 3'-UTR-WT luciferase plasmid containing the miR-875-5p binding sites and the COPS7A 3'-UTR-MUT plasmid containing the MUT binding sites were constructed. Cells $(5,637)$ were seeded into 96 -well plates $\left(1 \times 10^{4}\right.$ cells/well). Luciferase plasmids were co-transfected with miR-875-5p mimic or mimic-NC using Lipofectamine ${ }^{\circledR} 2000$ (Invitrogen; Thermo Fisher Scientific, Inc.). After incubated at $37^{\circ} \mathrm{C}$ for 48 h, cells $(5,637)$ were harvested. The Firefly and Renilla luciferase activities were analyzed using a Dual-Luciferase Reporter assay system (Promega Corporation) according to the manufacturer's instructions. The relative luciferase activity was normalized to Renilla luciferase activity. 
A

BCa stage II tissues (126 cases) vs. normal tissues (adjacent normal) (19 cases)

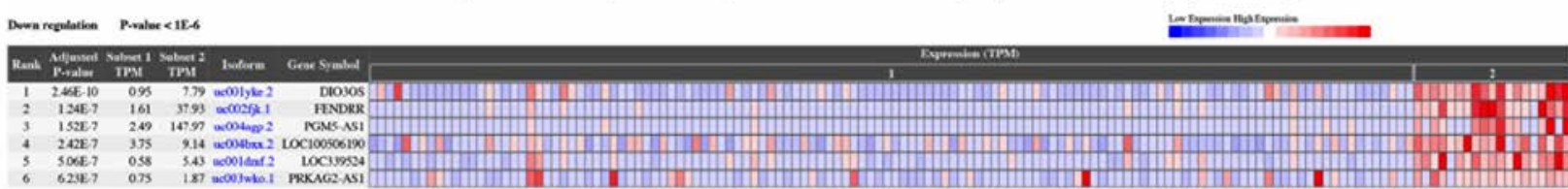

B

C
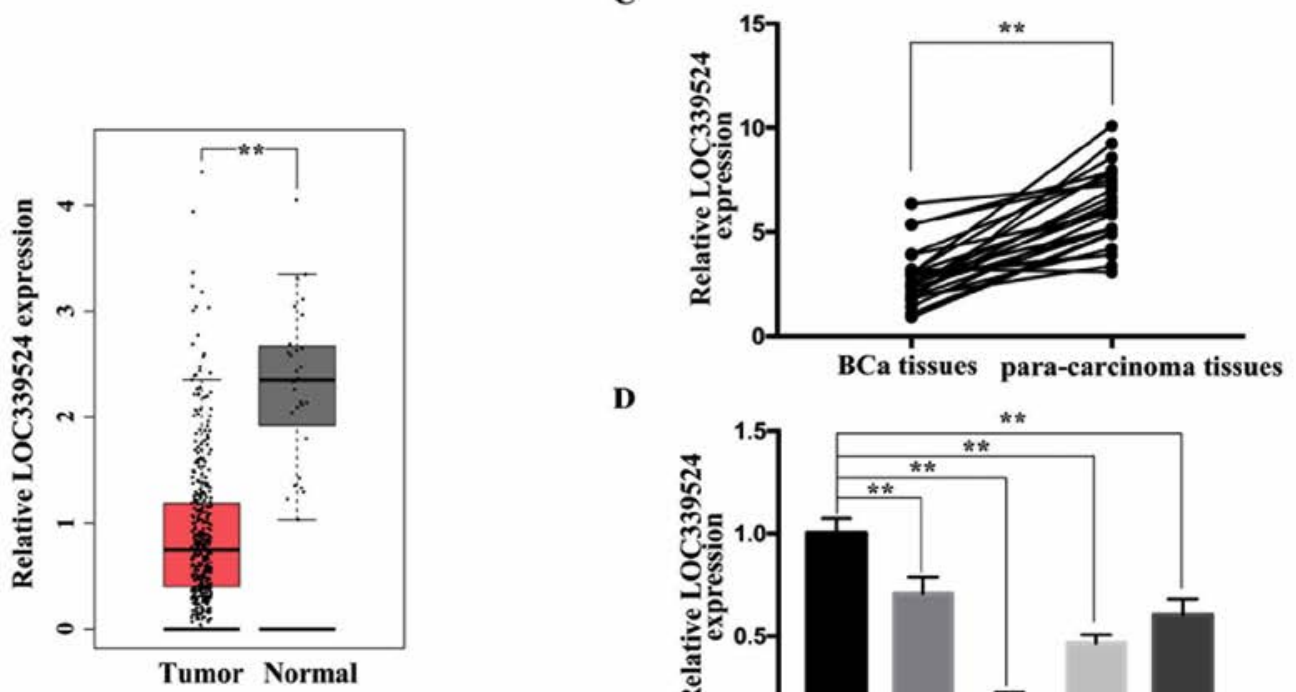

D

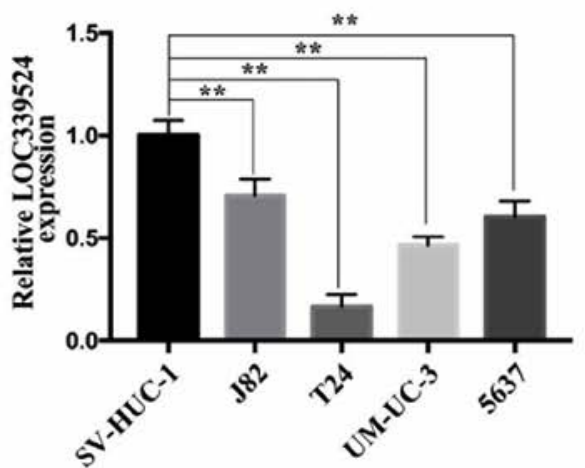

Figure 1. LOC339524 expression levels are downregulated in human BCa tissues and cell lines. (A) RNA-Seq data of LOC339524 expression in BCa cases obtained from The Cancer Genome Atlas were analyzed via the Cancer RNA-Seq Nexus. 1, BCa stage II tissues (126 cases); 2, adjacent normal tissues (19 cases). (B) Expression levels of LOC339524 in BCa and normal tissues were obtained from data in the Gene Expression Profiling Interactive Analysis database. (C) Expression levels of LOC339524 in (C) BCa tissues ( $\mathrm{n}=26)$ and para-carcinoma tissues $(\mathrm{n}=26)$ and (D) BCa cell lines and a normal bladder epithelial cell line (SV-HUC-1) were detected using reverse transcription-quantitative PCR. All data are presented as the mean $\pm \mathrm{SD}$. ${ }^{* *} \mathrm{P}<0.01$. BCa, bladder cancer; RNA-Seq, RNA-sequencing.

Western blotting. Cells were washed twice with PBS and total protein was extracted using RIPA lysis buffer (Beyotime Institute of Biotechnology) supplemented with PMSF protein inhibitor (Beyotime Institute of Biotechnology). The protein concentration was quantified using a standard BCA protein assay and $30 \mu \mathrm{g}$ of protein/lane was separated via 10\% SDS-PAGE. The separated proteins were subsequently transferred onto PVDF membranes (MilliporeSigma) and blocked with $5 \%$ non-fat milk at $26^{\circ} \mathrm{C}$ for $2 \mathrm{~h}$. The membranes were then incubated overnight at $4^{\circ} \mathrm{C}$ with the following primary antibodies: Rabbit anti-COPS7A (1:1,000; cat. no. ab124705; Abcam), rabbit anti-CDK2 (1:1,000; cat. no. ab32147; Abcam), rabbit anti-CDK4 (1:1,000; cat. no. ab108357; Abcam), rabbit anti-cyclin D2 (1:1,000; cat. no. ab230883; Abcam) and rabbit anti-GAPDH (1:2,500; cat. no. ab9485; Abcam). Following the primary antibody incubation, the membranes were incubated with a goat anti-rabbit IgG H\&L secondary antibody (1:5,000; cat. no. ab6721; Abcam) at room temperature for $2 \mathrm{~h}$ according to the protocol. The protein bands were visualized using an ECL system (Thermo Fisher Scientific, Inc.). GAPDH was used as the internal loading control.

Biotin pull-down assay. The biotin-labeled LOC339524 and miR-875-5p probe were synthesized by Changzhou Ruibo Bio-Technology Co., Ltd. The LOC339524 or miR-875-5p probe was incubated with streptavidin-coupled probe-bound DynaBeads (Invitrogen; Thermo Fisher Scientific, Inc.) at $4^{\circ} \mathrm{C}$ for $12 \mathrm{~h}$. Following incubation, the RNA complexes bound to the beads were eluted. RT-qPCR was used to detect the purified RNAs.

Statistical analysis. Data are presented as the mean \pm SD from at least three independent experiments. SPSS software (version 20.0; IBM Corp.) was used for statistical analysis. Comparisons among multiple groups were analyzed using a one-way ANOVA followed by a Tukey's post hoc test, while the statistical differences between two unpaired groups were analyzed using an unpaired Student's t-test. The statistical differences between tumor and adjacent non-tumor samples of the same individuals were analyzed using a paired Student's t-test. Correlations analysis were analyzed by the Pearson's correlation test. $\mathrm{P}<0.05$ was considered to indicate a statistically significant difference.

\section{Results}

LOC339524 is expressed at low levels in BCa tissues and cell lines. The expression profile of LOC339524 was screened from the CRN database. As shown in Fig. 1A, the expression levels of LOC339524 were downregulated in BCa tissues compared with 
A

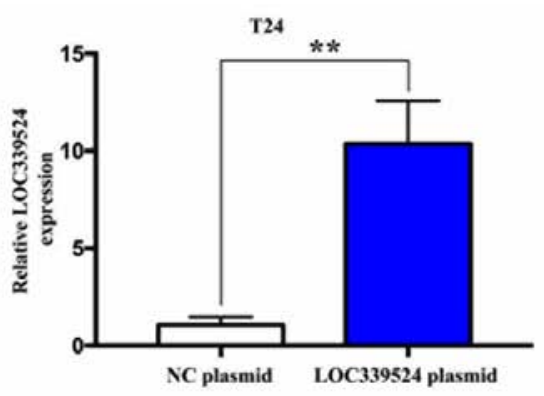

B

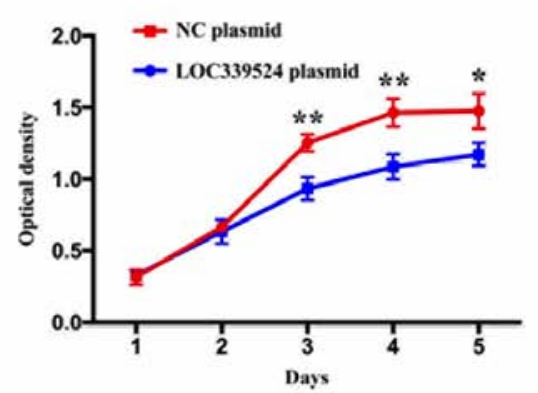

C

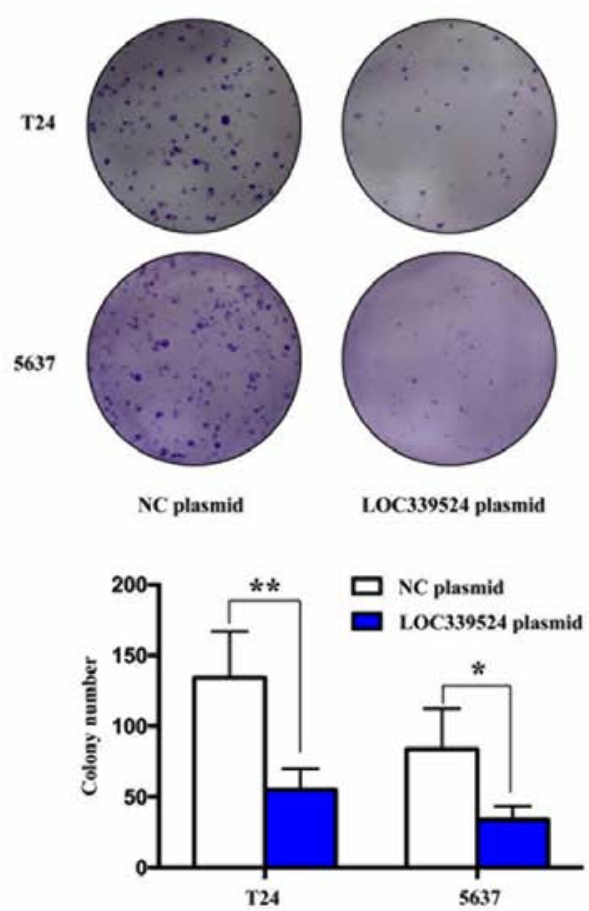

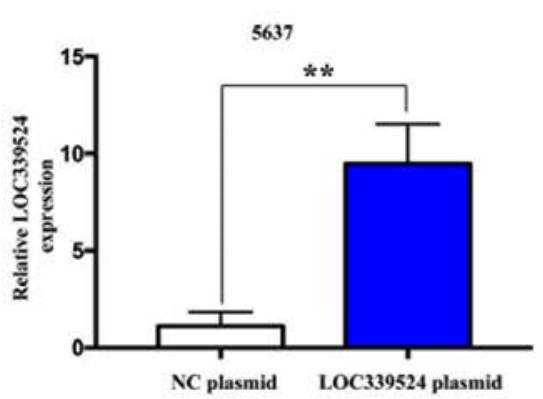

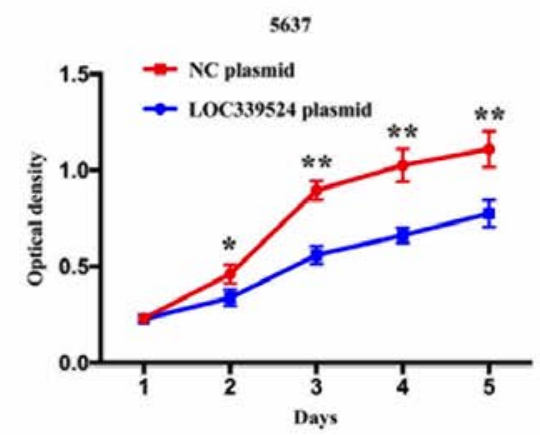

D
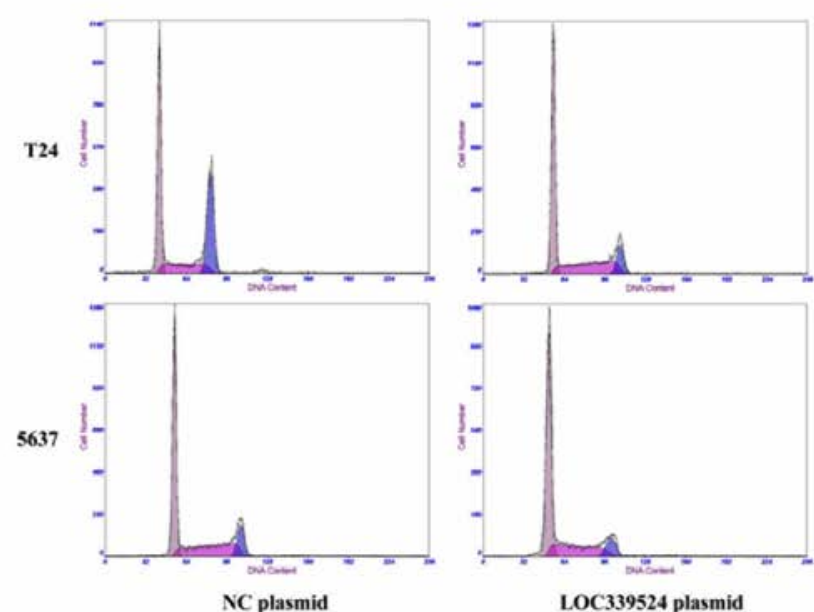

LOC339524 plasmid
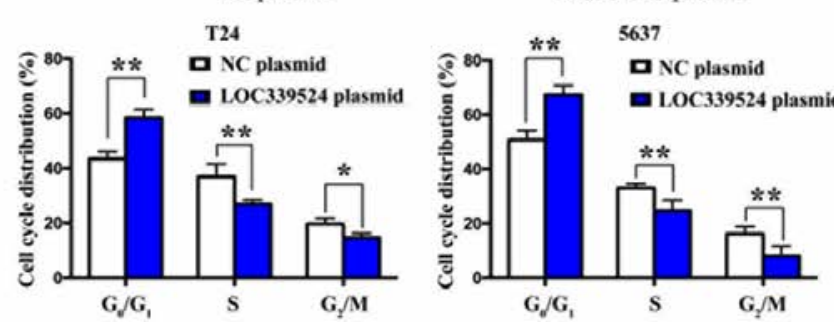

Figure 2. LOC339524 overexpression markedly inhibits BCa cell proliferation by promoting the G1 phase of the cell cycle. (A) Overexpression of LOC339524 in 5637 and T24 cells was verified using reverse transcription-quantitative PCR. (B) Cell Counting Kit- 8 assay was used to investigate cell proliferation following the overexpression of LOC339524. (C) Representative images of the colony formation assay following LOC339524 overexpression in 5637 and T24 cells. The number of colonies was counted and semi-quantified. (D) Flow cytometry was applied to detect the progression of the cell cycle. The percentage of $\mathrm{BCa}$ cells in the $\mathrm{G}_{0} / \mathrm{G}_{1}, \mathrm{~S}$ and $\mathrm{G}_{2} / \mathrm{M}$ phases are presented. All data are presented as the mean $\pm \mathrm{SD}$. ${ }^{*} \mathrm{P}<0.05$, ${ }^{* *} \mathrm{P}<0.01$. BCa, bladder cancer; $\mathrm{NC}$, negative control.

adjacent tissues. The data from the GEPIA database revealed the same trend (Fig. 1B). The expression of LOC339524 was then examined in 26 pairs of $\mathrm{BCa}$ and para-carcinoma tissues using RT-qPCR. The results revealed that LOC339524 expression levels were significantly downregulated in $\mathrm{BCa}$ tissues compared with those in para-carcinoma tissues (Fig. 1C). In addition, the expression levels of LOC339524 in four BCa cell lines (J82, T24, UM-UC-3 and 5637) were detected using RT-qPCR; the SV-HUC-1 cell line served as a control. Compared with the SV-HUC-1 cells, the expression levels of LOC339524 in the BCa cell lines were downregulated (Fig. 1D). Notably, 5637 and T24 cells exhibited the lowest expression levels of LOC339524 amongst the $\mathrm{BCa}$ cell lines.

IncRNA LOC339524 is closely associated with the proliferative ability of BCa cells. To investigate the role of LOC339524 in the 
A
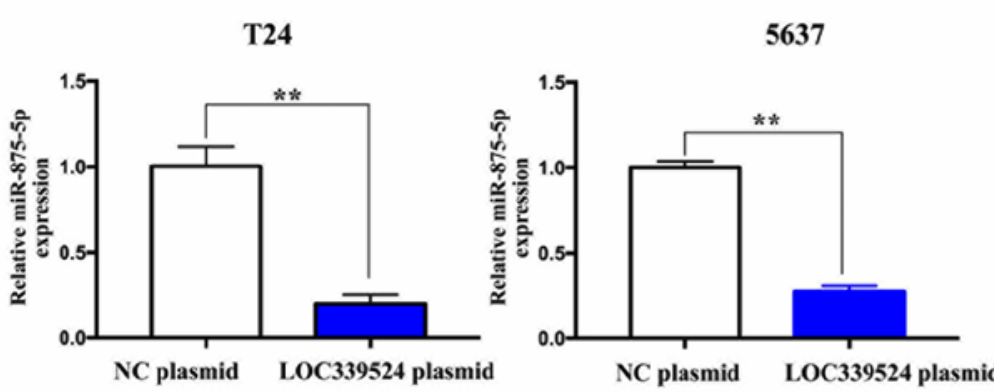

C

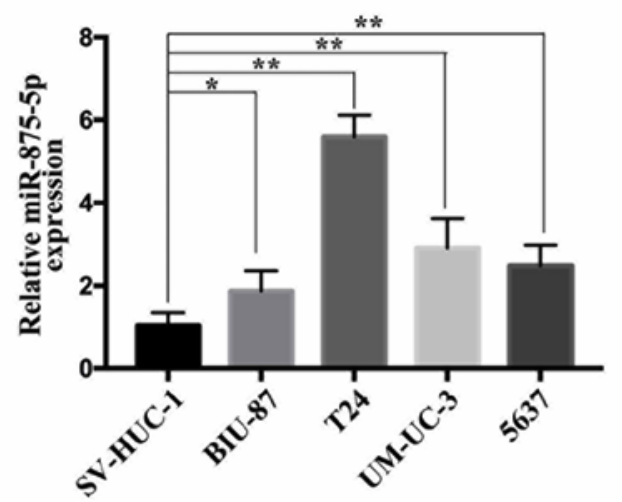

miR-875-5p 3' UGGACUAUUUUGACUCCAUAU 5' $1|1| 1|1| 1 \mid$

LOC339524 WT 5'... UUUGAACUACUGAGGUAUC... 3,

LOC339524 MUT 5'... UUUGAACUUGACUCCAUAC... 3'

G
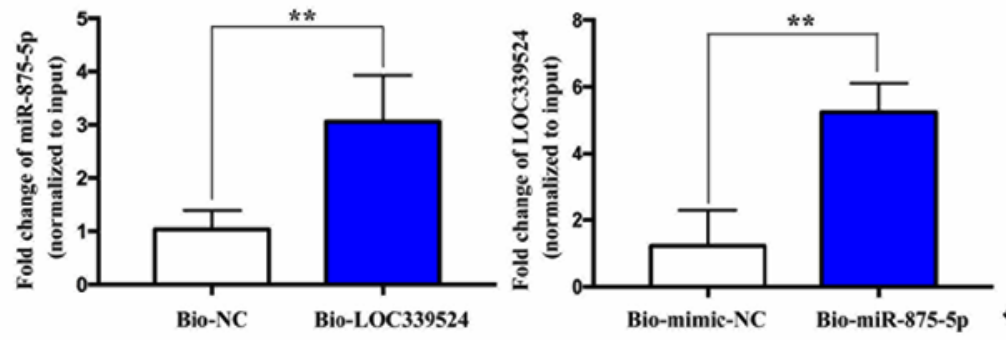

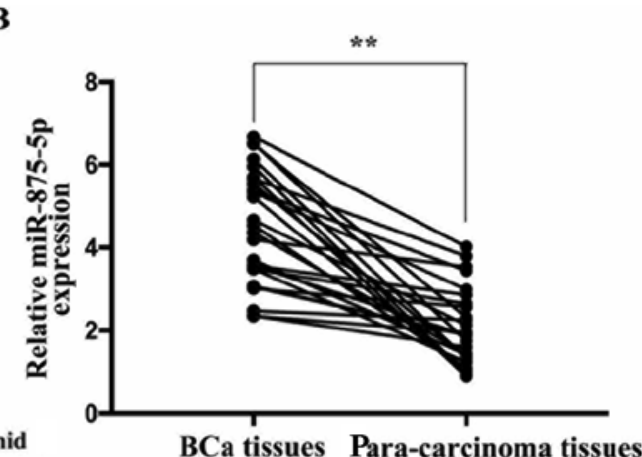

D

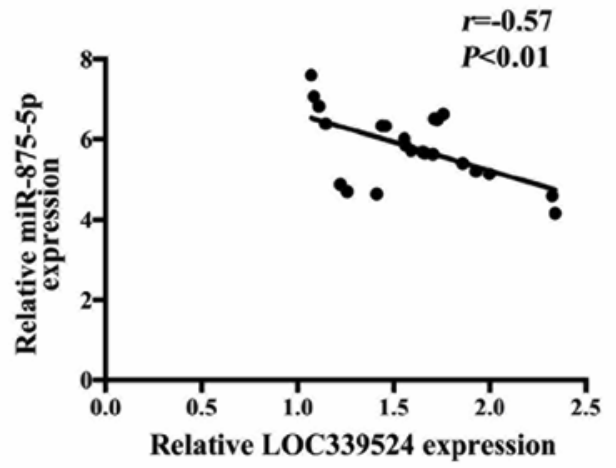

F

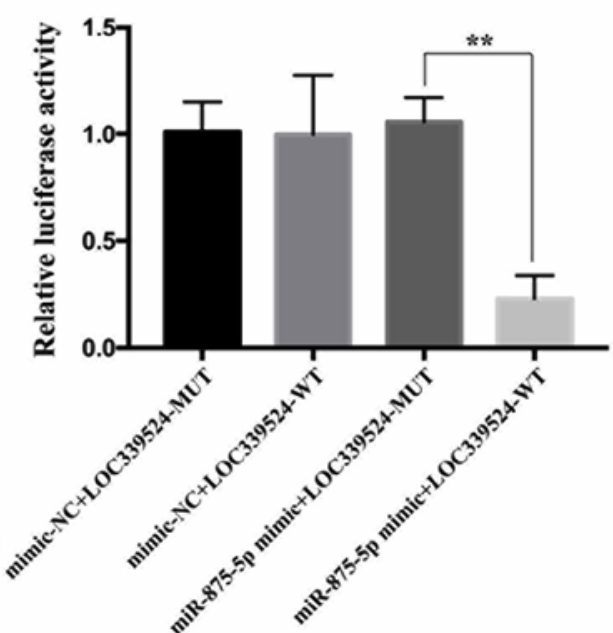

Figure 3. Screening and verification of miRNAs targeted by LOC339524. (A) RT-qPCR was used to detect the expression of miR-875-5p in cells overexpressing LOC339524. (B) Expression levels of miR-875-5p were significantly upregulated in BCa tissues compared with para-carcinoma tissues. (C) Expression levels of LOC339524 and miR-875-5p were negatively correlated in BCa tissues. (D) Expression levels of miR-875-5p in the BCa cell lines and a normal bladder epithelial cell line were analyzed using RT-qPCR. (E) WT and MUT seed sequences for miR-875-5p in LOC339524. (F) Dual luciferase reporter assays indicated that miR-875-5p overexpression notably decreased the relative luciferase activity in 5637 cells which were co-transfected with the LOC339524-WT vector. (G) Bio pull-down assays were used in 5637 cells. Co-precipitated LOC339524 or miR-875-5p was subsequently subjected to RT-qPCR analysis. All data are presented as the mean $\pm \mathrm{SD} .{ }^{*} \mathrm{P}<0.05,{ }^{* *} \mathrm{P}<0.01$. miRNA/miR, microRNA; BCa, bladder cancer; RT-qPCR, reverse transcription-quantitative PCR; NC, negative control; WT, wild-type; MUT, mutant; Bio, biotin.

proliferation of BCa cells, LOC339524 was successfully overexpressed in the 5637 and T24 cell lines (Fig. 2A). Cell proliferation was detected using a CCK-8 assay and the results indicated that LOC339524 overexpression suppressed the proliferative ability of the BCa cells (Fig. 2B). In addition, the colony formation ability was significantly suppressed when LOC339524 was overexpressed in both cell lines (Fig. 2C). Furthermore, cell cycle analysis indicated that the number of cells in the $G_{0} / G_{1}$ phase was increased following the overexpression of LOC339524, while that in the $S$ and $G_{2} / M$ phases was decreased compared with the NC plasmid group (Fig. 2D). Taken together, these findings indicated that LOC339524 may significantly inhibit the proliferation of $\mathrm{BCa}$ cells by blocking cell cycle progression.

LOC339524 serves as a ceRNA by binding to $\mathrm{miR}-875-5 \mathrm{p}$. The DIANA TOOLS database revealed that LOC339524 could possibly bind to miR-875-5p via complementary base pairing. The expression of miR-875-5p was detected by RT-qPCR after LOC339524 was overexpressed. The overexpression of LOC339524 significantly downregulated the expression of miR-875-5p compared with the NC plasmid group in both cell lines (Fig. 3A). Subsequently, the expression levels of 
$\mathbf{A}$
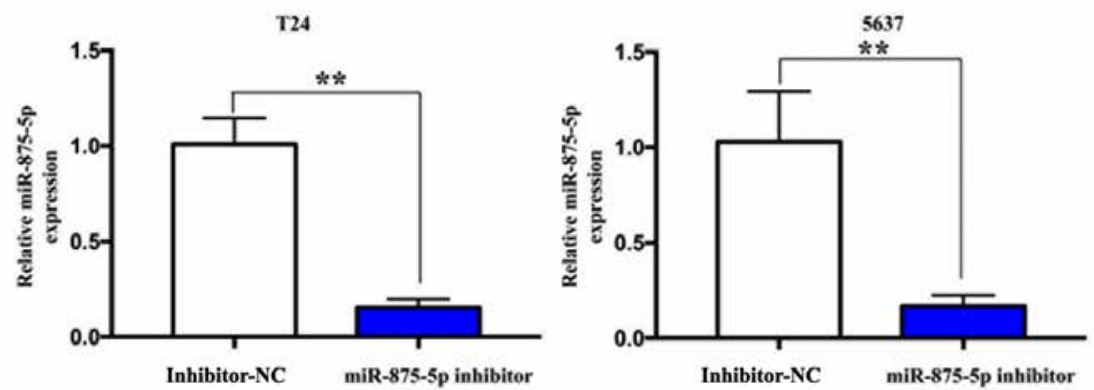

B

T24
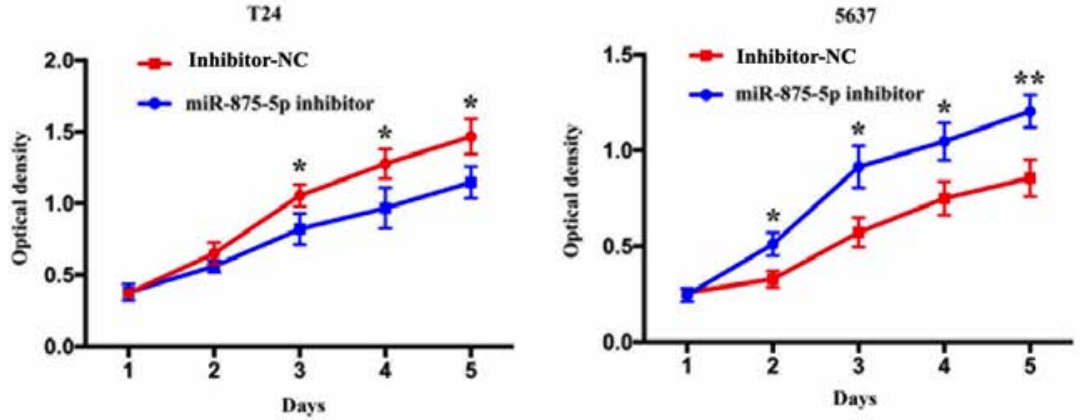

$\mathbf{C}$

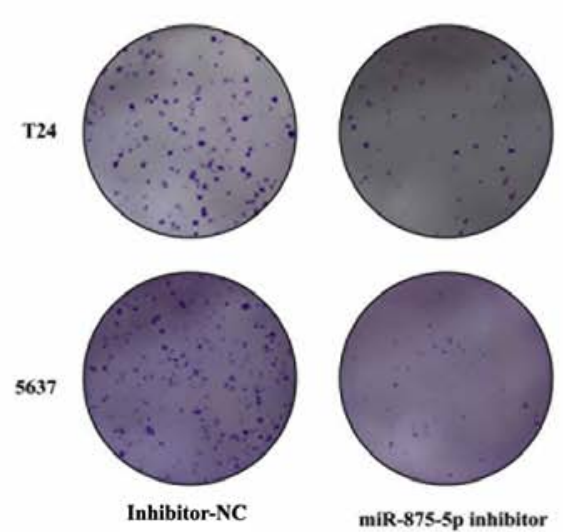

D
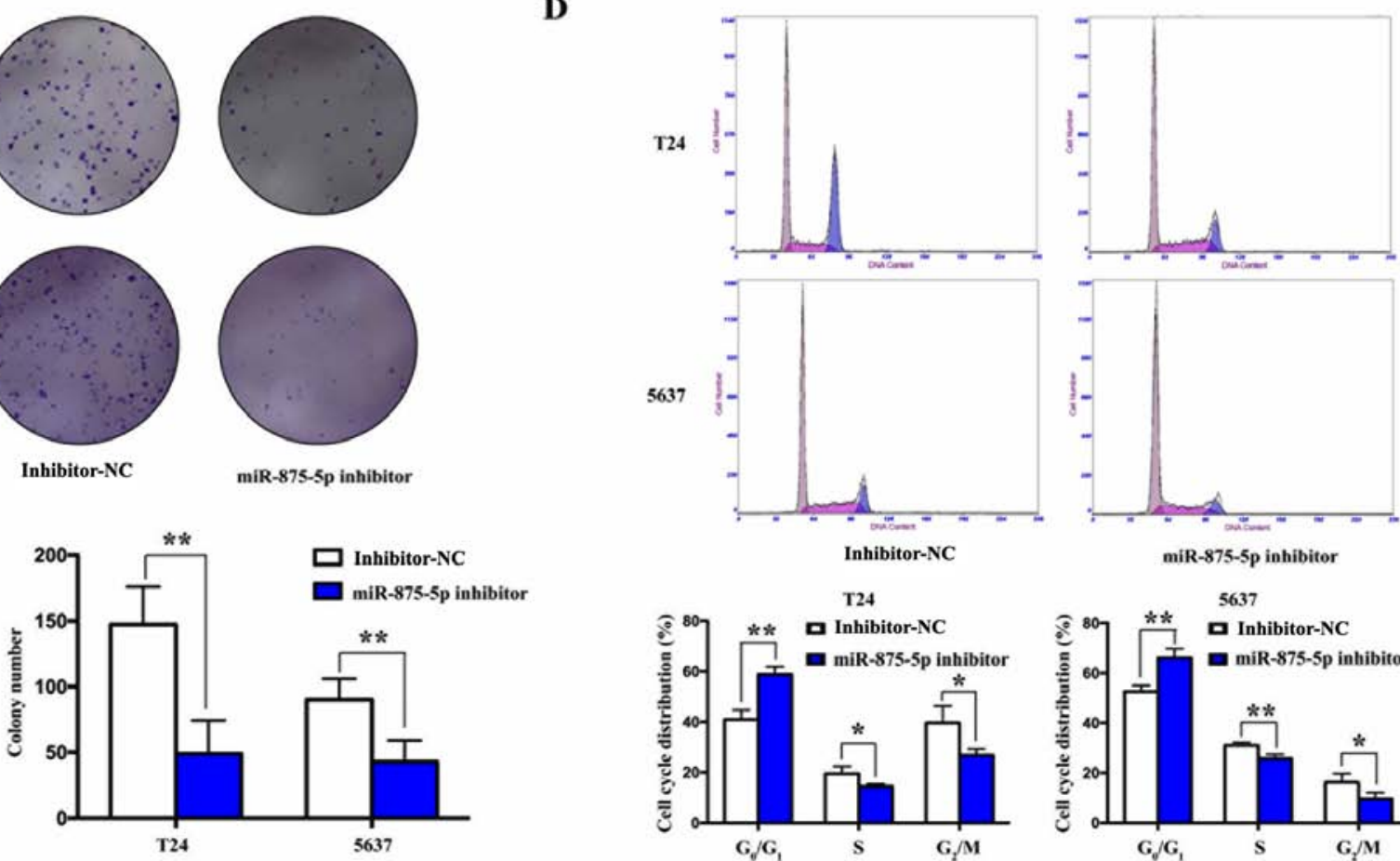

miR-875-5p inhibitor

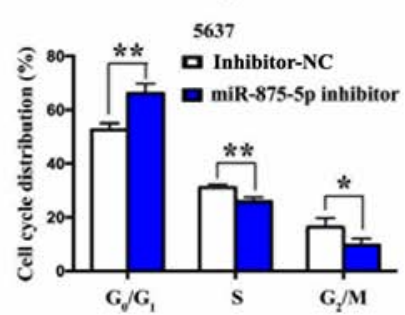

Figure 4. Knockdown of miR-875-5p significantly suppresses cell proliferation by accelerating the $\mathrm{G}_{1}$ phase of the cell cycle. (A) miR-875-5p expression levels were downregulated in 5637 and T24 cell lines following transfection with the miR-875-5p inhibitor. (B) Cell Counting Kit-8 assay was performed to assess the proliferation of 5637 and T24 cells following the knockdown of miR-875-5p. (C) Representative images of the colony formation of 5637 and T24 cells following the knockdown of miR-875-5p. The number of colonies was counted and semi-quantified. (D) Flow cytometry was used to detect the progression of the cell cycle. The percentage of bladder cancer cells in the $\mathrm{G}_{0} / \mathrm{G}_{1}, \mathrm{~S}$ and $\mathrm{G}_{2} / \mathrm{M}$ phases are presented. All data are presented as the mean $\pm \mathrm{SD}$. ${ }^{*} \mathrm{P}<0.05,{ }^{* *} \mathrm{P}<0.01$. miR, microRNA; NC, negative control.

miR-875-5p were detected in $26 \mathrm{BCa}$ and para-carcinoma tissues using RT-qPCR. miR-875-5p expression was found to significantly upregulated in BCa samples compared with para-carcinoma samples (Fig. 3B). In addition, the expression levels of miR-875-5p in BCa cell lines were significantly upregulated compared with those in the SV-HUC-1 cells (Fig. 3C). Furthermore, the expression levels of LOC339524 and miR-875-5p were negatively correlated with each other in BCa tissues (Fig. 3D). Thus, LOC339524 was hypothesized to function as a sponge for miR-875-5p.

The fluorescent reporter plasmids, LOC339524-WT and LOC339524-MUT, containing the miR-875-5p binding site, were constructed (Fig. 3E). miR-875-5p overexpression significantly decreased the relative luciferase activity 
A

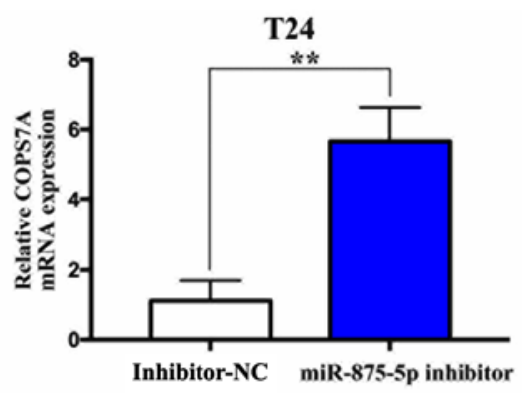

B

miR-875-5p 3' UGGACUAUUUUGACUCCAUAU 5 ,

COPS7A mRNA WT 5 '... GUGGGGAAGGGAAGGGUAUA.... 3 ,

COPS7A mRNA MUT 5 '... GUGGGGAAGGGAAGCCAUAU... 3'

C
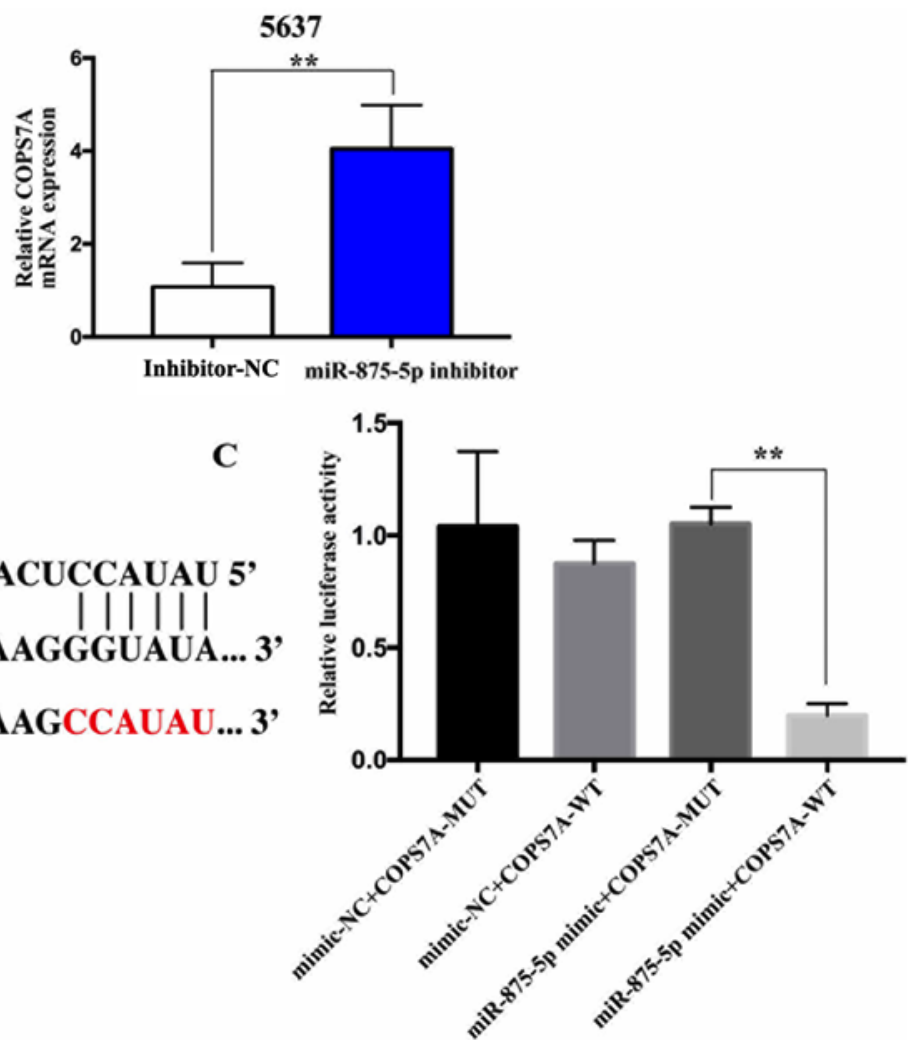

Figure 5. Screening and validation of miR-875-5p target genes. (A) Following the knockdown of miR-875-5p in the 5637 and T24 cell lines, the mRNA expression levels of COPS7A were notably upregulated. (B) WT and MUT seed sequence for miR-875-5p in the 3'-untranslated region of COPS7A. (C) Dual luciferase reporter assays suggested that transfection with COPS7A-WT plasmid and miR-875-5p notably decreased the relative luciferase activity in 5637 cells compared with transection with the COPS7A-MUT plasmid and miR-875-5p. All data are presented as the mean \pm SD. ${ }^{* *} \mathrm{P}<0.01$. miR, microRNA; COPS7A, COP9 signalosome subunit 7A; NC, negative control; WT, wild-type; MUT, mutant.

following co-transfection with LOC339524-WT in 5637 cells, whereas miR-875-5p overexpression exerted no significant effects on the relative luciferase activity of the cells following co-transfection with LOC339524-MUT (Fig. 3F). These findings indicated that LOC339524 may directly bind to miR-875-5p. A biotin pull-down assay was then conducted to investigate the endogenous interaction between LOC339524 and miR-875-5p. The results indicated that LOC339524 and miR-875-5p were significantly enriched in the bio-miR-875-5p and bio-LOC339524 groups, respectively, compared with the bio-NC/mimic-NC group (Fig. 3G).

Knockdown of miR-875-5p suppresses the proliferation of $B C$ a cells. To examine the effects of miR-875-5p expression on the proliferative ability of $\mathrm{BCa}$ cells, miR-875-5p expression was knocked down in BCa 5637 and T24 cells via transfection with a miR-875-5p inhibitor. Transfection with miR-875-5p inhibitor significantly downregulated the expression levels of miR-875-5p compared with the inhibitor-NC-transfected cells (Fig. 4A). Cell proliferation was then detected using CCK-8 and colony formation assays. The results revealed that the proliferation and colony formation ability of the cells were significantly inhibited following the knockdown of miR-875-5p compared with the inhibitor-NC transfected cells (Fig. 4B and C). In addition, cell cycle analysis revealed that, following the knockdown of miR-875-5p, the number of cells in the $S$ and $G_{2} / M$ phases decreased, while that in the $\mathrm{G}_{0} / \mathrm{G}_{1}$ phase increased (Fig. 4D). These results indicated that the knockdown of miR-875-5p may inhibit the proliferation of $\mathrm{BCa}$ cells.

miRWalk and TargetScan databases were screened to predict the target genes of miR-875-5p, and COPS7A was predicted as the candidate target gene. The mRNA expression levels of COPS7A were found to be upregulated following the inhibition of miR-875-5p in 5637 and T24 cells compared with the inhibitor-NC group (Fig. 5A). Subsequently, the luciferase reporter plasmids, COPS7A 3'-UTR-WT and COPS7A 3'-UTR-MUT, containing the WT or MUT miR-875-5p binding site, were respectively constructed (Fig. 5B). The results revealed that the 5637 cells co-transfected with miR-875-5p mimic and the COPS7A 3'-UTR-WT plasmid exhibited a significant decrease in the relative luciferase activity compared with cells co-transfected with miR-875-5p mimic and the COPS7A 3'-UTR-MUT. However, the overexpression of miR-875-5p did not affect the relative luciferase activity of 5637 cells co-transfected with the COPS7A 3'-UTR-MUT plasmid. These findings indicated that miR-875-5p may directly bind to COPS7A mRNA (Fig. 5C).

COPS7A expression is downregulated in BCa and the downregulation of LOC339524 occurs due to the hypermethylation of its promoter. The mRNA expression levels of COPS7A were analyzed in $26 \mathrm{BCa}$ and para-carcinoma tissues using RT-qPCR. COPS7A mRNA expression levels were discovered to be significantly downregulated in BCa tissues compared with para-carcinoma tissues (Fig. 6A). Furthermore, a significant 
A

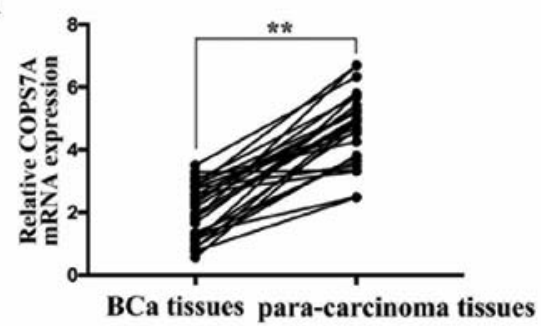

C
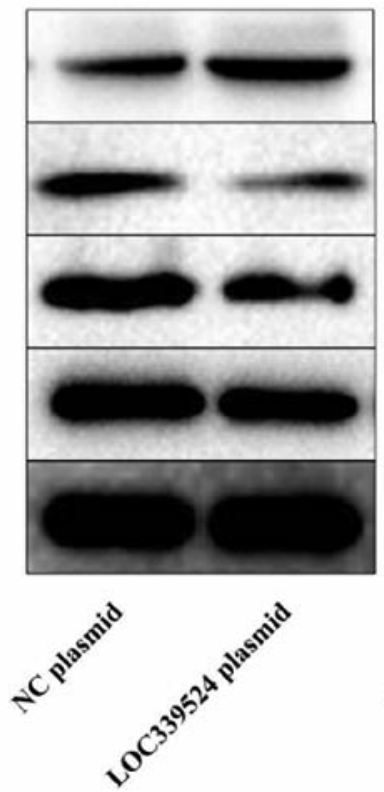
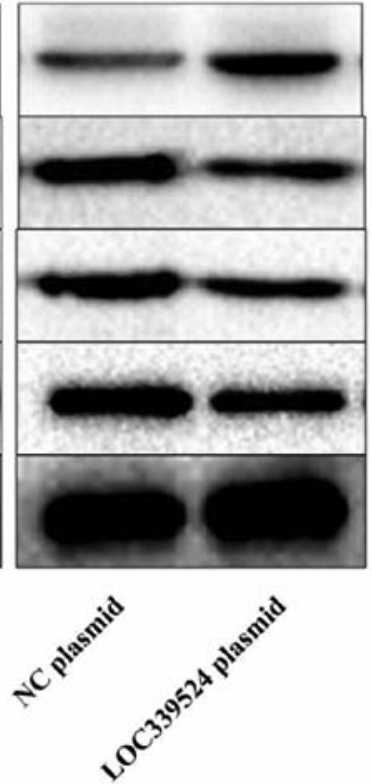

B

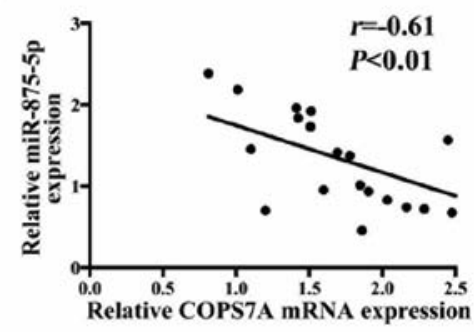

D

COPS7A

CDK2

CDK4

Cyclin D2

GAPDH

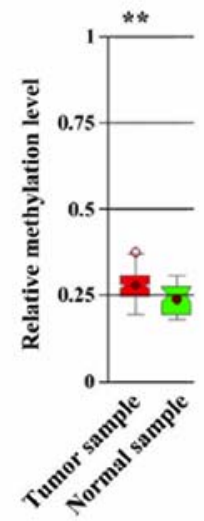

E

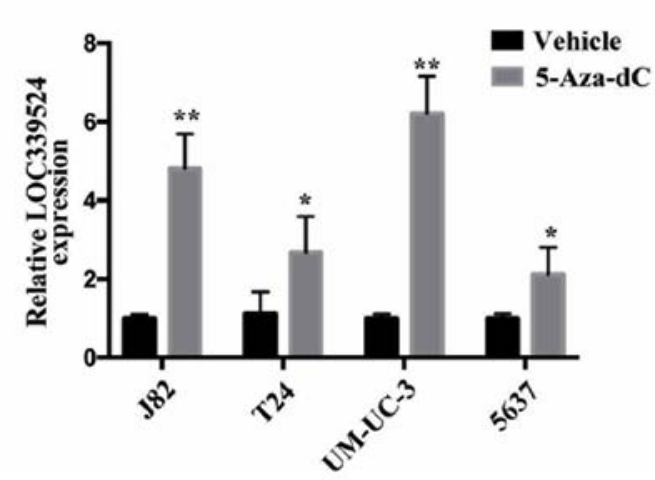

Figure 6. COPS7A is expressed at low levels in BCa, and the downregulation of LOC339524 occurs due to the increased methylation of its promoter. (A) Expression of COPS7A was markedly decreased in BCa tissues compared with in para-carcinoma tissues. (B) Expression of COPS7A mRNA and miR-875-5p were negatively correlated in BCa tissues. (C) Following LOC339524 overexpression in 5637 and T24 cell lines, the expression of COPS7A was notably increased at the protein level, the expression of CDK2, CDK4 and Cyclin D2 proteins was significantly reduced. (D) MethHC database was used to determine the DNA methylation status of LOC339524 in BCa. (E) Expression of LOC339524 in BCa cell lines following treatment with 5-Aza-CdR. All data are presented as the mean $\pm \mathrm{SD} .{ }^{*} \mathrm{P}<0.05,{ }^{* *} \mathrm{P}<0.01$. miR, microRNA; $\mathrm{BCa}$, bladder cancer; COPS7A, COP9 signalosome subunit 7A; NC, negative control; 5-Aza-CdR, 5-Aza-2'-deoxycytidine.

negative correlation was identified between the expression of COPS7A mRNA and miR-875-5p (Fig. 6B). Following LOC339524 overexpression in 5637 and T24 cells, the protein expression levels of COPS7A were notably increased, while the expression of CDK2, CDK4 and cyclin D1 proteins was notably decreased (Fig. 6C). These results indicated that LOC339524 may serve as a ceRNA in a network involving miR-875-5p and COPS7A. The MethHC database revealed the degree of methylation of the LOC339524 promoter in $\mathrm{BCa}$ (Fig. 6D). The results suggested that the promoter was notably hypermethylated in $\mathrm{BCa}$ and that the low expression of LOC339524 may be associated with the methylation status. Following treatment with $5 \mu \mathrm{l}$ 5-Aza-2'-deoxycytidine (Sigma-Aldrich; Merck KGaA; $2 \mu \mathrm{mol} / \mathrm{l}$ ), the expression levels of LOC339524 were significantly upregulated in BCa cell lines compared with the vehicle-treated cells (Fig. 6E).

\section{Discussion}

$\mathrm{BCa}$ is the commonest malignant cancer of the urinary system. Surgery and chemotherapy are the most common methods used to treat $\mathrm{BCa}$ (18). Despite advancements being made in the treatment of $\mathrm{BCa}$, the mortality rate of patients with advanced-stage $\mathrm{BCa}$ remains high, indicating resistance to treatment (19). To improve the treatment efficacy for $\mathrm{BCa}$, novel therapeutic strategies and targets are required. A multitude of lncRNAs have been identified to serve as critical regulators of the development of BCa (20). Dai et al (21) reported that 
lncRNA integrin subunit $\beta 1$ (ITGB1) was upregulated in $\mathrm{BCa}$ tissues and affected the malignant progression of $\mathrm{BCa}$ by regulating the expression of miR-10a. The expression levels of ITGB1 were also found to be associated with the pathological stage of BCa and were suggested to be useful as a novel predictor of prognosis. Fang et al (22) demonstrated that lncRNA distal-less homeobox 6 antisense 1 (DXL6-AS1) was highly expressed in $\mathrm{BC}$ tissues and acted as a sponge for miR-223. DXL6-AS1 has been shown to reduce miR-223 expression, thereby enhancing $\mathrm{BCa}$ cell proliferation and invasion. Yang et al (23) reported that lncRNA long intergenic non-protein coding RNA 319 (LINC00319) was upregulated in BCa tissues. LINC00319 enhanced cell proliferation and invasion by functioning as a ceRNA to sponge miR-4492, which attenuated its downstream target, reactive oxygen species modulator 1. Zhuang et al (24) found that lncRNA gastric cancer-associated lncRNA1 (GClnc1) promoted the proliferation, migration and invasiveness of $\mathrm{BCa}$ cells by regulating the lin-28 homolog B/let-7a/Myc signaling pathway. These findings indicated that lncRNAs may be used as potential biomarkers and targets for BCa. According to current research (24), the majority of lncRNAs in BCa serve as oncogenes. To the best of our knowledge, lncRNAs functioning as tumor suppressor genes in $\mathrm{BCa}$ are rarely reported.

In the present study, abnormally expressed lncRNAs were screened in BCa using the CRN database and lncRNA LOC339524 was selected for subsequent analysis. The roles of LOC339524 in types of cancer have not been previously reported, to the best of the authors' knowledge. The expression levels of LOC339524 were found to be markedly downregulated in $\mathrm{BCa}$ tissues compared with para-carcinoma tissues. Similarly, compared with SV-HUC-1 cells, lncRNA LOC339524 expression was low in BCa cells, particularly in 5637 and T24 cells. Thus, 5637 and T24 cells were selected for use in subsequent analyses. The in vitro experiments identified that LOC339524 inhibited the proliferation of $\mathrm{BCa}$ cells. Previous studies have demonstrated that lncRNAs can serve as ceRNAs that sponge miRNAs, thereby affecting the expression of target genes mediated by miRNAs and regulating the malignant activity of cancer cells $(25,26)$. Therefore, we hypothesized that LOC339524 may affect the biological behavior of $\mathrm{BCa}$ cells through a ceRNA mechanism. The present study confirmed that LOC339524 and miR-875-5p could bind to each other, as demonstrated by bioinformatics analysis and dual luciferase reporter assays. Wang et al (27) found that miR-875-5p was upregulated in non-small cell lung cancer tissues, and attenuated SATB homeobox 2 expression to the promote proliferation and invasion of non-small cell lung cancer cells. Notably, miR-875-5p has been reported to exert tumor-promoting effects in lung cancer (27). However, to the best of our knowledge, the role of miR-875-5p in BCa has not been studied to date. The results of the present study also revealed that miR-875-5p expression was significantly upregulated in $\mathrm{BCa}$ tissues and cells. The results of the current study demonstrated that miR-875-5p served as an oncogene in BCa. COPS7A belongs to the COP9 signalosome complex (CSN) complex (28). CSN expression has been shown to control cell cycle progression and to be associated with carcinogenesis (29). COPS7A expression was also found to be downregulated in gastric cancer and suppressed gastric cancer cell proliferation by inactivating the $\mathrm{NF}-\kappa \mathrm{B}$ signaling pathway (30). In the present study, COPS7A expression was found to be downregulated in $\mathrm{BCa}$ tissues. To the best of our knowledge, the present study was the first to demonstrate that COPS7A is a direct target gene of miR-875-5p via bioinformatics analysis and dual luciferase reporter assays. Furthermore, it was identified that the promoter of LOC339524 in BCa was markedly hypermethylated, and the decreased expression of LOC339524 was associated with promoter hypermethylation.

However, there are several limitations in this study. The expression of LOC339524 were analyzed using limited sample sizes. The functional experiments in the current study were conducted at the cellular level and only proved the function of LOC339524 in BCa cells instead of animals. Further studies are needed to confirm the expression of LOC339524 in a large sample size and observe the roles of LOC339524 in animal experiments.

In conclusion, the findings of the present study demonstrated that the expression levels of LOC339524 were downregulated in $\mathrm{BCa}$ tissues and cells. It was also found that LOC339524 suppressed the proliferation of BCa cells in vitro. Therefore, LOC339524 was proposed to function as a ceRNA that promotes the expression of COPS7A by binding and sponging miR-875-5p.

\section{Acknowledgements}

Not applicable.

\section{Funding}

No funding was received.

\section{Availability of data and materials}

The datasets used and/or analyzed during the current study are available from the corresponding author on reasonable request.

\section{Authors' contributions}

BW and $\mathrm{XH}$ designed the experiments; HX, RY and HC performed the experiments; $\mathrm{BW}$ and $\mathrm{XH}$ analyzed and interpreted the data and drafted the manuscript. All authors read and approved the final manuscript. BW and $\mathrm{XH}$ confirm the authenticity of all the raw data.

\section{Ethics approval and consent to participate}

The present study was approved by the Ethics Committee of The Third Affiliated Hospital, Army Medical University (Chongqing, China). All patients were informed of the study protocol and signed written informed consent forms.

\section{Patient consent for publication}

Not applicable.

\section{Competing interests}

The authors declare that they have no competing interests. 


\section{References}

1. Sung H, Ferlay J, Siegel RL, Laversanne M, Soerjomataram I Jemal A and Bray F: Global Cancer Statistics 2020: GLOBOCAN Estimates of incidence and mortality worldwide for 36 cancers in 185 countries. CA Cancer J Clin 71: 209-249, 2021.

2. Abdolmaleki F, Ghafouri-Fard S, Taheri M and Omrani DM: P21-Associated ncRNA DNA damage-activated expression in bladder cancer. Klin Onkol 32: 277-280, 2019.

3. Jiang D, Zhang Y, Yang L, Lu W, Mai L, Guo H and Liu X: Long noncoding RNA HCG22 suppresses proliferation and metastasis of bladder cancer cells by regulation of PTBP1. J Cell Physiol 2: $1711-1722,2019$.

4. Hu J, Shan Y, Ma J, Pan Y, Zhou H, Jiang L and Jia L: IncRNA ST3Gal6-AS1/ST3Gal6 axis mediates colorectal cancer progression by regulating alpha-2,3 sialylation via PI3K/Akt signaling. Int J Cancer 145: 450-460, 2019.

5. Bai J, Xu J, Zhao J and Zhang R: Downregulation of lncRNA AWPPH inhibits colon cancer cell proliferation by downregulating GLUT-1. Oncol Lett 18: 2007-2012, 2019.

6. Li Y, Zhao L, Zhao P and Liu Z: Long non-coding RNA LINC00641 suppresses non-small-cell lung cancer by sponging miR-424-5p to upregulate PLSCR4. Cancer Biomark 26: 79-91, 2019.

7. Lee YR, Kim G, Tak WY, Jang SY, Kweon YO, Park JG, Lee HW, Han YS, Chun JM, Park SY and Hur K: Circulating exosomal noncoding RNAs as prognostic biomarkers in human hepatocellular carcinoma. Int J Cancer 144: 1444-1452, 2019.

8. Li J, Wang W, Xia P, Wan L, Zhang L, Yu L, Wang L, Chen X, $\mathrm{Xiao} \mathrm{Y}$ and $\mathrm{Xu} \mathrm{C}$ : Identification of a five-lncRNA signature for predicting the risk of tumor recurrence in patients with breast cancer. Int J Cancer 143: 2150-2160, 2018.

9. Yao F, Wang Q and $\mathrm{Wu} \mathrm{Q}$ : The prognostic value and mechanisms of lncRNA UCA1 in human cancer. Cancer Manag Res 11: 7685-7696, 2019.

10. Zhang Y, Liao G, Bai J, Zhang X, Xu L, Deng C, Yan M, $\mathrm{Xie} A$, Luo $\mathrm{T}$, Long $\mathrm{Z}$, et al: Identifying cancer driver lncRNAs bridged by functional effectors through integrating multi-omics data in human cancers. Mol Ther Nucleic Acids 17: 362-373, 2019.

11. Dai G, Huang C, Yang J, Jin L, Fu K, Yuan F, Zhu J and Xue B: lncRNA SNHG3 promotes bladder cancer proliferation and metastasis through miR-515-5p/GINS2 axis. J Cell Mol Med 24: 9231-9243, 2020.

12. Li Y, Shi B, Dong F, Zhu X, Liu B and Liu Y: lncRNA KCNQ1OT1 facilitates the progression of bladder cancer by targeting miR-218-5p/HS3ST3B1. Cancer Gene Ther 28 212-220, 2021.

13. Livak KJ and Schmittgen TD: Analysis of relative gene expression data using real-time quantitative PCR and the 2(-Delta Delta C(T)) method. Methods 25: 402-408, 2001.

14. Li JR, Sun CH, Li W, Chao RF, Huang CC, Zhou XJ and Liu CC: Cancer RNA-Seq Nexus: A database of phenotype-specific transcriptome profiling in cancer cells. Nucleic Acids Res 44: D944-D951, 2016.

15. Tang Z, Li C, Kang B, Gao G, Li C and Zhang Z: GEPIA: A web server for cancer and normal gene expression profiling and interactive analyses. Nucleic Acids Res 45: W98-W102, 2017.

16. Paraskevopoulou MD, Georgakilas G, Kostoulas N, Reczko M, Maragkakis M, Dalamagas TM and Hatzigeorgiou AG: DIANA-lncBase: Experimentally verified and computationally predicted microRNA targets on long non-coding RNAs. Nucleic Acids Res 41: D239-D245, 2013.
17. Huang HY, Li J, Tang Y, Huang YX, Chen YG, Xie YY, Zhou ZY, Chen XY, Ding SY, Luo MF, et al: MethHC 2.0: Information repository of DNA methylation and gene expression in human cancer. Nucleic Acids Res 49: D1268-D1275, 2021.

18. Yuan S, Luan X, Han G, Guo K, Wang S and Zhang X: LINC01638 lncRNA mediates the postoperative distant recurrence of bladder cancer by upregulating ROCK2. Oncol Lett 18: 5392-5398, 2019.

19. Hu X, Feng H, Huang H, Gu W, Fang Q, Xie Y, Qin C and Hu X: Downregulated long noncoding RNA PART1 inhibits proliferation and promotes apoptosis in bladder cancer. Technol Cancer Res Treat 18: 1533033819846638, 2019.

20. Lyu L, Xiang W, Zhu JY, Huang T, Yuan JD and Zhang CH: Integrative analysis of the lncRNA-associated ceRNA network reveals lncRNAs as potential prognostic biomarkers in human muscle-invasive bladder cancer. Cancer Manag Res 11: 6061-6077, 2019.

21. Dai L, Chai CM, Shen TY, Tian Y, Shang ZQ and Niu YJ: lncRNA ITGB1 promotes the development of bladder cancer through regulating microRNA-10a expression. Eur Rev Med Pharmacol Sci 23: 6858-6867, 2019.

22. Fang C, Xu L, He W, Dai J and Sun F: Long noncoding RNA DLX6-AS1 promotes cell growth and invasiveness in bladder cancer via modulating the miR-223-HSP90B1 axis. Cell Cycle 18: 3288-3299, 2019.

23. Yang Y, Zhang F, Huang H, Xie Z, Huang W, Xie $\mathrm{H}$ and Wang F: Long noncoding RNA LINC00319 regulates ROMO1 expression and promotes bladder cancer progression via miR-4492/ROMO1 axis. J Cell Physiol 4: 3768-3775, 2019.

24. Zhuang C, Ma Q, Zhuang C, Ye J, Zhang F and Gui Y: lncRNA GClnc1 promotes proliferation and invasion of bladder cancer through activation of MYC. FASEB J 33: 11045-11059, 2019.

25. Peng $\mathrm{H}$ and $\mathrm{Li} \mathrm{H}$ : The encouraging role of long noncoding RNA small nuclear RNA host gene 16 in epithelial-mesenchymal transition of bladder cancer via directly acting on miR-17-5p/metalloproteinases 3 axis. Mol Carcinog 58: 1465-1480, 2019.

26. Peng Y, Leng W, Duan S and Hong M: Long noncoding RNA BLACAT1 is overexpressed in hepatocellular carcinoma and its downregulation suppressed cancer cell development through endogenously competing against hsa-miR-485-5p. Biomed Pharmacother 116: 109027, 2019.

27. Wang J, Lu Y, Ding H, Gu T, Gong C, Sun J, Zhang Z, Zhao Y and Ma C: The miR-875-5p inhibits SATB2 to promote the invasion of lung cancer cells. Gene 644: 13-19, 2018.

28. Davidsson J and Johansson B: Methylation and expression analyses of Pallister-Killian syndrome reveal partial dosage compensation of tetrasomy $12 p$ and hypomethylation of gene-poor regions on 12p. Epigenetics 11: 194-204, 2016.

29. Yoshida A, Yoneda-Kato N, Panattoni M, Pardi R and Kato JY: CSN5/Jab1 controls multiple events in the mammalian cell cycle. FEBS Lett 584: 4545-4552, 2010

30. Zheng J, Zhang $\mathrm{H}$, Ma R, Liu $\mathrm{H}$ and Gao P: Long non-coding RNA KRT19P3 suppresses proliferation and metastasis through COPS7A-mediated NF- $\kappa \mathrm{B}$ pathway in gastric cancer. Oncogene 45: 7073-7088, 2019.

This work is licensed under a Creative Commons Attribution-NonCommercial-NoDerivatives 4.0 International (CC BY-NC-ND 4.0) License. 